\title{
Research Article Spectral Analysis of Sampled Signals in the Linear Canonical Transform Domain
}

\author{
Bing-Zhao Li and Tian-Zhou Xu \\ School of Mathematics, Beijing Institute of Technology, Beijing 100081, China \\ Correspondence should be addressed to Bing-Zhao Li, li_bingzhao@bit.edu.cn
}

Received 11 September 2011; Revised 11 December 2011; Accepted 13 December 2011

Academic Editor: Zhan Shu

Copyright (C) 2012 B.-Z. Li and T.-Z. Xu. This is an open access article distributed under the Creative Commons Attribution License, which permits unrestricted use, distribution, and reproduction in any medium, provided the original work is properly cited.

The spectral analysis of uniform or nonuniform sampling signal is one of the hot topics in digital signal processing community. Theories and applications of uniformly and nonuniformly sampled one-dimensional or two-dimensional signals in the traditional Fourier domain have been well studied. But so far, none of the research papers focusing on the spectral analysis of sampled signals in the linear canonical transform domain have been published. In this paper, we investigate the spectrum of sampled signals in the linear canonical transform domain. Firstly, based on the properties of the spectrum of uniformly sampled signals, the uniform sampling theorem of two dimensional signals has been derived. Secondly, the general spectral representation of periodic nonuniformly sampled one and two dimensional signals has been obtained. Thirdly, detailed analysis of periodic nonuniformly sampled chirp signals in the linear canonical transform domain has been performed.

\section{Introduction}

The sampling process is one of the fundamental concepts of digital signal processing, which serves as a bridge between the continuous physical signals and discrete signals. The sampling process can be marked as uniform sampling and nonuniform sampling according to the sampling offsets. Theories and applications of uniformly and nonuniformly sampled one and two-dimensional signals in traditional Fourier domain have been well studied [13]. The spectral analysis of uniform and nonuniform samples has also been investigated in the Fourier domain [4-6]. However, most of the theories and methods derived in the literature are only suitable for bandlimited signals of Fourier transform (FT) domain, they will derive the wrong (or at least suboptimal) conclusions about signals and systems that not bandlimited in the Fourier domain [7]. For example, signals such as gravity waves, broadband chirp signals, and radar and sonar signals may behave as nonbandlimited in the Fourier 
domain. It is therefore worthwhile and interesting to explore the new nonstationary signal processing tools and derive novel properties of nonbandlimited signals of the Fourier domain in the new transform domain.

With the efforts of the scientists of signal processing community, many useful tools such as the wavelet transform, the fractional Fourier transform [8], the Hilbert-Huang transform [9], and the linear canonical transform (LCT) have been proposed to process nonstationary signals. The LCT, which was introduced during the 1970s with four parameters [10], has been proven to be one of the most powerful tools for nonstationary signal processing. The signal processing transforms, such as the Fourier transform (FT), the fractional Fourier transform (FrFT), the Fresnel transform, and the scaling operations are all special cases of the LCT [8]. The well-known concepts associated with the traditional Fourier transform, such as the uncertainty principles [11], the convolution and product theorem $[12,13]$, the Hilbert transform [14-16], and the Poisson summation formula [17] are well studied and extended in the LCT domain. The expansion of the classical uniform or nonuniform sampling theorem for the band-limited or time-limited signal in the LCT domain has recently been studied [18-20]. Recently, the authors of [21,22] address the multichannel sampling problems and their novel results can be looked as the generalization of the well-known sampling theorem for the LCT and FT domain. These sampling theorems establish the fact that a band-limited or time-limited continuous signal in the LCT domain can be completely reconstructed by a set of equidistantly spaced signal samples, but so far none of the research papers covering the spectral analysis of sampled signals in the LCT domain have been published. Therefore, exploring the spectral properties for sampled signals in the LCT domain is worthwhile and interesting.

Focusing on the spectral analysis of uniformly or nonuniformly sampled signals in the LCT domain, this paper investigates the spectrum of signals based on the uniform and periodic nonuniform samples in the LCT domain. The paper is organized as follows. The Preliminary is proposed in Section 2. In Section 3, the uniform sampling theorem of twodimensional LCT is derived based on the definition of LCT and the traditional sampling theorem. The spectral analysis of one and two dimensional periodic nonuniform sampling signals is derived in Section 4. Section 5 is the conclusion of the paper.

\section{The Preliminary}

\subsection{The Linear Canonical Transform (LCT)}

The LCT with parameters $\left(\begin{array}{ll}a & b \\ c & d\end{array}\right)$ of a signal $f(t)$ is defined as [8]:

$$
L_{f}^{A}(u)=L_{f}^{A}[f(t)](u)= \begin{cases}\sqrt{\frac{1}{j 2 \pi b}} \int_{-\infty}^{+\infty} f(t) e^{j(1 / 2)\left[(a / b) t^{2}-(2 / b) t u+(d / b) u^{2}\right]} d t, & b \neq 0, \\ \sqrt{d} e^{j(1 / 2) c d u^{2}} f(d u), & b=0\end{cases}
$$

where

$$
A \equiv\left(\begin{array}{ll}
a & b \\
c & d
\end{array}\right), \quad \operatorname{det}(A)=a d-b c=1
$$


The LCT of two-dimensional signal $f(x, y)$ is defined as [16]:

$$
\begin{aligned}
L_{f}^{A, B}\{f(x, y)\}= & L_{f}^{A, B}(u, v)=\iint_{-\infty}^{+\infty} f(x, y) K_{A, B}(x, y, u, v) d x d y \\
& =\left\{\begin{array}{c}
\frac{1}{2 \pi j} \sqrt{\frac{1}{b_{1} b_{2}}} \cdot e^{j\left[\left(d_{1} u^{2} / 2 b_{1}\right)+\left(d_{2} v^{2} / 2 b_{2}\right)\right]} \\
\times \iint_{-\infty}^{+\infty} e^{-j\left[\left(u x / b_{1}\right)+\left(v y / b_{2}\right)\right]} \cdot e^{j\left[\left(a_{1} x^{2} / 2 b_{1}\right)+\left(a_{2} y^{2} / 2 b_{2}\right)\right]} f(x, y) d x d y, \\
b_{1} b_{2} \neq 0,|A|=|B|=1 \\
\sqrt{d_{1} d_{2}} \cdot e^{j\left[\left(c_{1} d_{1} u^{2}+c_{2} d_{2} v^{2} / 2\right)\right]} f\left(d_{1} u, d_{2} v\right), \quad b_{1}{ }^{2}+b_{2}{ }^{2}=0,
\end{array}\right.
\end{aligned}
$$

where

$$
\begin{gathered}
K_{A, B}(x, y, u, v)=K_{A}(x, u) K_{B}(y, v), \quad K_{A}(x, u)=\sqrt{\frac{1}{2 \pi j b_{1}}} \cdot e^{j\left(d_{1} u^{2} / 2 b_{1}\right)} e^{-j\left(u x / b_{1}\right)} e^{j\left(a_{1} x^{2} / 2 b_{1}\right)} ; \\
K_{B}(y, v)=\sqrt{\frac{1}{2 \pi j b_{2}}} \cdot e^{j\left(d_{2} v^{2} / 2 b_{2}\right)} e^{-j\left(v y / b_{2}\right)} e^{j\left(a_{2} y^{2} / 2 b_{2}\right)}, \quad A=\left(\begin{array}{ll}
a_{1} & b_{1} \\
c_{1} & d_{1}
\end{array}\right), \quad B=\left(\begin{array}{ll}
a_{2} & b_{2} \\
c_{2} & d_{2}
\end{array}\right) .
\end{gathered}
$$

In this paper we restrict ourselves to the class of the LCT with real parameters, that is, the parameters in matrix $A, B$ are real numbers.

\subsection{The Sampling Theorem of One-Dimensional Signal in the LCT Domain}

It is shown in $[18,19]$ that if a signal is nonbandlimited in the Fourier domain, then it can be bandlimited in the LCT domain. Therefore, the common sampling theorem in Fourier domain may not suitable for these nonbandlimited signals. In order to solve these problems, the uniform sampling theorems for one-dimensional signal in the LCT domain have been derived as shown below $[18,19]$.

Lemma 2.1. Assume a signal $f(t)$ bandlimited to $\Omega_{A}$ in the LCT domain with parameter $A=\left(\begin{array}{ll}a & b \\ c & d\end{array}\right)$ and $b>0$; then the sampling theorem for signal $f(t)$ can be expressed as:

$$
f(t)=e^{-j(a / 2 b) t^{2}} \sum_{n=-\infty}^{+\infty} f(n T) e^{j(a / 2 b)(n T)^{2}} \frac{\sin \left[\Omega_{A}(t-n T) / b\right]}{\Omega_{A}(t-n T) / b}
$$

where $T$ is the sampling period and satisfies $T=\pi b / \Omega_{A}$; and the Nyquist rate of sampling theorem associated with the LCT is $f_{s}=\Omega_{A} / \pi b$.

Proof. see $[18,19]$. 


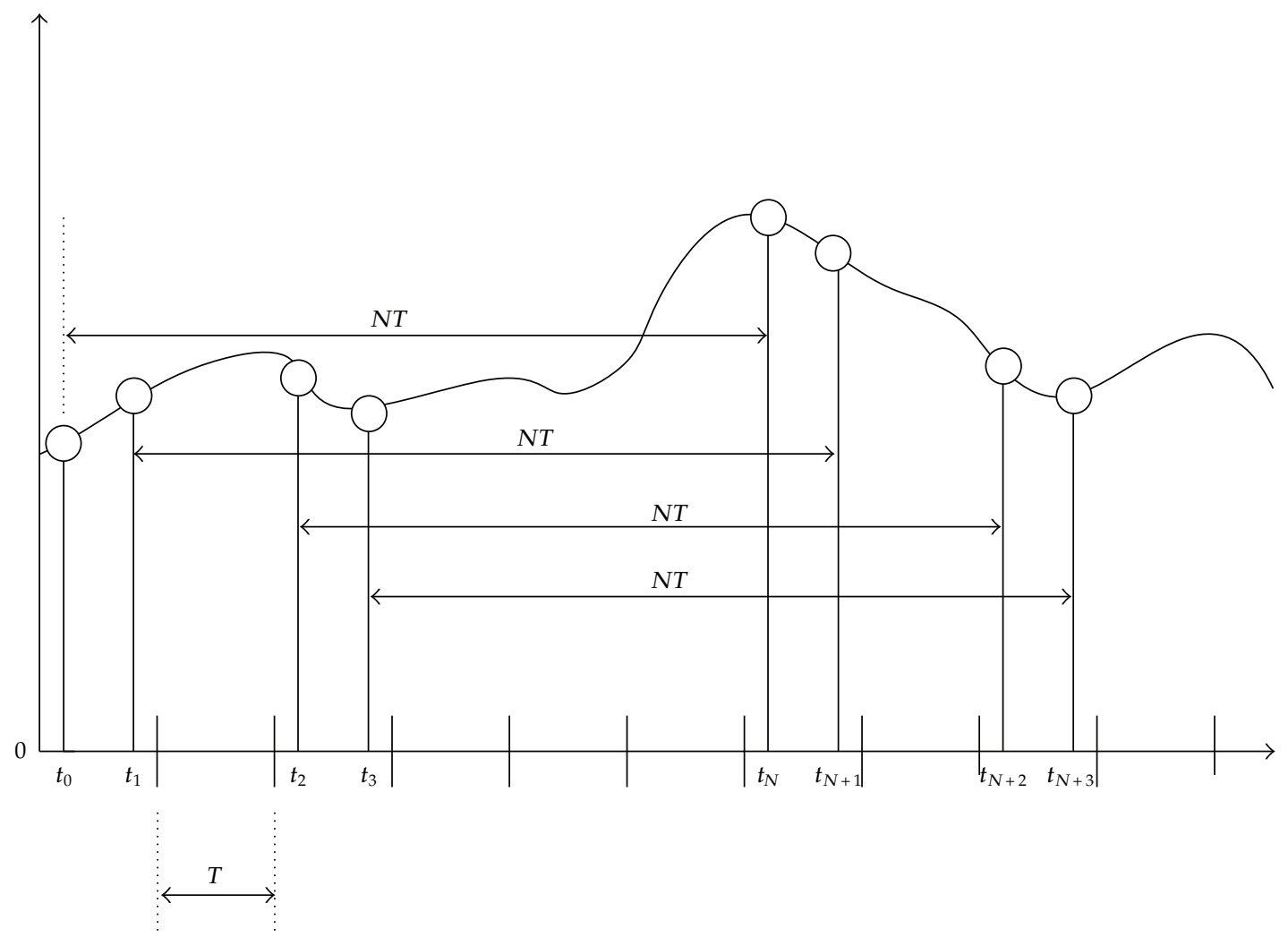

Figure 1: The recurrent nonuniform sampling model [5].

\subsection{The Periodic Nonuniform Sampling Model}

The periodic nonuniform sampling [4-6, 21-23], which also named as recurrent nonuniform sampling, arises in a broad range of applications. For example [4], we might consider converting a continuous time signal to a discrete time signal using a series of $A / D$ converters, and each is operating at a rate lower than the Nyquist rate such that the average sampling rate is equal to the Nyquist rate. This may be beneficial in applications where high-rate $A / D$ converters are required. Typically, the cost and complexity of a converter will increase (more than linearly) with the rate. In such cases, we can benefit from converting a continuous time signal to a discrete time signal using $N A / D$ converters [23], each is operating at one $N$ th of the Nyquist rate. Since the converters are typically not synchronized, the resulting discrete time signal is a combination of $N$ sequences of uniform samples. Thus, the resulting discrete time signal corresponds to recurrent nonuniform samples of the continuous time signal.

In this form of sampling, the sampling points are divided into several groups of points. The groups have a recurrent period, which is denoted by $N T$ and equal to $N$ times the Nyquist period. Each period consists of $N$ nonuniform sampling points. Denoting the points in one period by $t_{n}, n=0,1, \ldots, N-1$, the complete set of sampling points are $t_{n}+k N T$, $n=0,1,2, \ldots, N-1, k \in Z$. Periodic nonuniform samples can be regarded as a combination of $N$ sequences of uniform samples taken at one $N$ th of the Nyquist rate. An example of this periodic nonuniform sampling distribution is depicted in Figure 1 [5]. 
Besides the one-dimensional recurrent nonuniform sampling model, in real applications we often meet the multidimensional nonuniform sampling. Jenq investigates the twodimensional periodic nonuniform sampling model and obtains a perfect spectral reconstruction method in the Fourier domain $[5,6]$, Feuer and Coodwin introduce the multidimensional recurrent nonuniform sampling model in [4] and derive the reconstruction methods based on the filterbanks. It is shown that a specific example of this situation arises when one utilizes multiple identical digital cameras on the same scene [4].

The signal recovery from one or multidimensional recurrent nonuniform sampling points are well studied in the Fourier domain; however, there are no paper published about the spectral analysis from recurrent nonuniform sampling points in the LCT domain. It is worthwhile and interesting to investigate the spectral analysis and reconstruction in the LCT domain.

\section{The Uniform Sampling Theorems}

\subsection{The Uniform Sampling Theorem of Two-Dimensional Signals}

This section focuses on the uniform sampling theorem for two-dimensional signals in the LCT domain. Let $f(x, y)$ be an analog two-dimensional signal with its continuous LCT $L_{f}^{A, B}(u, v)$ bandlimited to $\left(\Omega_{x}, \Omega_{y}\right)$ in the LCT domain. In other words, $L_{f}^{A, B}(u, v)=0$, when $|u| \geq \Omega_{x}$ and $|v| \geq \Omega_{y}$.

Suppose $f_{s}(x, y)$ is obtained by sampling the signal $f(x, y)$ at uniformly spaced grids $T_{x}$ and $T_{y}, T_{x}$ and $T_{y}$ are uniform sampling period on $X$ and $Y$-axis, respectively, we have

$$
f_{s}(x, y)=\sum_{n=-\infty}^{\infty} \sum_{k=-\infty}^{\infty} f(x, y) \delta\left(x-n T_{x}\right) \delta\left(y-k T_{y}\right)
$$

Then, the spectrum of these samples function $f_{s}(x, y)$ in the LCT domain can be shown in the following theorem.

Theorem 3.1. Suppose $f_{s}(x, y)$ is obtained by sampling the continuous signal $f(x, y)$ at uniformly spaced grids $T_{x}$ and $T_{y}$, then the spectrum of these uniformly samples can be represented as:

$$
\begin{aligned}
D L_{f}^{A, B}(u, v)= & \frac{1}{T_{x} T_{y}} e^{j\left[\left(d_{1} u^{2} / 2 b_{1}\right)+\left(d_{2} v^{2} / 2 b_{2}\right)\right]} \\
& \times \sum_{k=-\infty}^{+\infty} \sum_{l=-\infty}^{+\infty} L_{f}^{A, B}\left(u-\frac{2 \pi k}{T_{x}} b_{1}, v-\frac{2 \pi l}{T_{y}} b_{2}\right) \\
& \times e^{-j\left(d_{1} / 2 b_{1}\right)\left(u-\left(2 \pi k / T_{x}\right) b_{1}\right)^{2}} e^{-j\left(d_{2} / 2 b_{2}\right)\left(v-\left(2 \pi l / T_{y}\right) b_{2}\right)^{2}}
\end{aligned}
$$


Proof. Applying the LCT to both sides of (3.1), we obtain

$$
\begin{aligned}
D L_{f}^{A, B}(u, v)= & \iint_{-\infty}^{+\infty} f_{s}(x, y) K_{A, B}(x, y, u, v) d x d y \\
= & \frac{1}{2 \pi j} \sqrt{\frac{1}{b_{1} b_{2}}} \cdot e^{j\left[\left(d_{1} u^{2} / 2 b_{1}\right)+\left(d_{2} v^{2} / 2 b_{2}\right)\right]} \\
& \cdot \iint_{-\infty}^{+\infty} e^{-j\left[\left(u x / b_{1}\right)+\left(v y / b_{2}\right)\right]} e^{j\left[\left(a_{1} x^{2} / 2 b_{1}\right)+\left(a_{2} y^{2} / 2 b_{2}\right)\right]} \\
& \times \sum_{n=-\infty}^{+\infty} \sum_{k=-\infty}^{+\infty} f(x, y) \delta\left(x-n T_{x}\right) \delta\left(y-k T_{y}\right) d x d y .
\end{aligned}
$$

Rearranging (3.3) and using the identity $\sum_{k=-\infty}^{+\infty} \delta\left(x-k T_{x}\right) \sum_{k=-\infty}^{+\infty} \delta\left(x-k T_{x}\right)=$ $\left(1 / T_{x}\right) \sum_{k=-\infty}^{+\infty} e^{j\left(2 k \pi x / T_{x}\right)}\left(1 / T_{y}\right) \sum_{k=-\infty}^{+\infty} e^{j\left(2 k \pi y / T_{y}\right)},(3.3)$, can be rewritten as

$$
\begin{aligned}
D L_{f}^{A, B}= & \frac{1}{2 \pi j} \sqrt{\frac{1}{b_{1} b_{2}}} \cdot e^{j\left[\left(d_{1} u^{2} / 2 b_{1}\right)+\left(d_{2} v^{2} / 2 b_{2}\right)\right]} \\
& \cdot \iint_{-\infty}^{+\infty} f(x, y) e^{-j\left[\left(u x / b_{1}\right)+\left(v y / b_{2}\right)\right]} e^{j\left[\left(a_{1} x^{2} / 2 b_{1}\right)+\left(a_{2} y^{2} / 2 b_{2}\right)\right]} \\
& \times\left\{\frac{1}{T_{x}} \sum_{k=-\infty}^{+\infty} e^{\left(j 2 \pi k x / T_{x}\right)} \cdot \frac{1}{T_{y}} \sum_{l=-\infty}^{+\infty} e^{\left(j 2 \pi l y / T_{y}\right)}\right\} d x d y \\
= & \frac{1}{2 \pi j} \frac{1}{T_{x} T_{y}} \sqrt{\frac{1}{b_{1} b_{2}}} \cdot e^{j\left[\left(d_{1} u^{2} / 2 b_{1}\right)+\left(d_{2} v^{2} / 2 b_{2}\right)\right]} \\
& \cdot \sum_{k=-\infty}^{+\infty} \sum_{l=-\infty}^{+\infty} \iint_{-\infty}^{+\infty} f(x, y) e^{-j\left[\left(\left(u-\left(2 \pi k / T_{x}\right) b_{1}\right) x / b_{1}\right)+\left(v-\left(2 \pi k / T_{y}\right) b_{2} y / b_{2}\right)\right]} e^{j\left[\left(a_{1} x^{2} / 2 b_{1}\right)+\left(a_{2} y^{2} / 2 b_{2}\right)\right]} .
\end{aligned}
$$

The final result can be obtained by the definition of the LCT.

From (3.2), the spectra of two-dimensional uniform sampling signal in the LCT domain can be seen as the repetition of $L_{f}^{A, B}(u, v) e^{-j\left(d_{1} / 2 b_{1}\right) u^{2}} e^{-j\left(d_{2} / 2 b_{2}\right) v^{2}}$, and these repetitions do not overlap if we chose the sampling interval $T_{x} \leq \pi b_{1} / \Omega_{x}, T_{y} \leq \pi b_{2} / \Omega_{y}$. In order to single out just one copy, we apply a low-pass filter to $D L_{f}^{A, B}(u, v)$.

$$
H(u, v)= \begin{cases}T_{x} T_{y}, & |u| \leq \Omega_{x},|v| \leq \Omega_{y} \\ 0, & \text { others. }\end{cases}
$$

So the original signal $f(x, y)$ can be derived by the inverse LCT transform of $D L_{f}^{A, B}(u$, $v) H(u, v)$. The reconstruction formula is presented in the following theorem. 
Theorem 3.2. suppose $f(x, y)$ be an analog two-dimensional signal with its continuous LCT $L_{f}^{A, B}(u, v)$ bandlimited to $\left(\Omega_{x}, \Omega_{y}\right)$ in the LCT domain. Then the original signal $f(x, y)$ can be reconstructed by the following uniform sampling formula

$$
\begin{aligned}
f(x, y)= & e^{-j\left(a_{1} / 2 b_{1}\right) x^{2}-j\left(a_{2} / 2 b_{2}\right) y^{2}} \\
& \times \sum_{n=-\infty}^{+\infty} \sum_{k=-\infty}^{+\infty} f\left(n T_{x}, k T_{y}\right) e^{j\left(a_{1} / 2 b_{1}\right)\left(n T_{x}\right)^{2}+j\left(a_{2} / 2 b_{2}\right)\left(k T_{y}\right)^{2}} \\
& \cdot \frac{\sin \left[\Omega_{x}\left(x-n T_{x}\right) / b_{1}\right]}{\Omega_{x}\left(x-n T_{x}\right) / b_{1}} \frac{\sin \left[\Omega_{y}\left(y-k T_{y}\right) / b_{2}\right]}{\Omega_{y}\left(y-k T_{y}\right) / b_{2}}
\end{aligned}
$$

where $T_{x}, T_{y}$ is the sampling period in the $X$-axis and $Y$-axis, respectively, and satisfies $T_{x} \leq$ $\pi b_{x} / \Omega_{x}, T_{y} \leq \pi b_{y} / \Omega_{y}$.

Proof. From the definition of $D L_{f}^{A, B}(u, v) H(u, v)$, the original signal can be derived by the inverse LCT transform of $D L_{f}^{A, B}(u, v) H(u, v)$. In other words, the original signal can be rewritten as:

$$
\begin{aligned}
f(x, y)= & L^{A^{-1}, B^{-1}}\left\{D L_{f}^{A, B}(u, v) H(u, v)\right\}(x, y) \\
= & \frac{1}{T_{x} T_{y}} \iint_{-\infty}^{+\infty} K_{A^{-1}}(u, x) K_{B^{-1}}(v, y) \\
& \times \iint_{-\infty}^{+\infty} f\left(x_{n, k}, y_{n, k}\right) K_{A}\left(x_{n, k}, u\right) K_{B}\left(y_{n, k}, v\right) H(u, v) d x_{n, k} d y_{n, k} d u d v \\
= & \int_{-\infty}^{+\infty} f\left(x_{n, k}, y_{n, k}\right) \\
& \times \int_{-\infty}^{+\infty} K_{A^{-1}}(u, x) K_{A}\left(x_{n, k}, u\right) K_{B^{-1}}(v, y) K_{B}\left(y_{n, k}, v\right) H(u, v) d u d v d x_{n, k} d y_{n, k} \\
= & \left(\frac{1}{2 \pi}\right)^{2} \frac{1}{b_{1} b_{2}} e^{-\left(a_{1} / 2 b_{1}\right) x^{2}-\left(a_{2} / 2 b_{2}\right) y^{2}} \\
& \times \iint_{-\infty}^{+\infty} f\left(x_{n, k}, y_{n, k}\right) e^{\left(a_{1} / 2 b_{1}\right) x_{n, k^{2}}} e^{\left(a_{2} / 2 b_{2}\right) y_{n, k^{2}}} \\
& \times \iint_{-\infty}^{+\infty} e^{\left(1 / b_{1}\right) u\left(x-x_{n, k}\right)} e^{\left(1 / b_{2}\right) v\left(y-y_{n, k}\right)} H(u, v) d u d v d x_{n, k} d y_{n, k} .
\end{aligned}
$$


Because

$$
\begin{aligned}
& \iint_{-\infty}^{+\infty} e^{\left(1 / b_{1}\right) u\left(x-x_{n, k}\right)} e^{\left(1 / b_{2}\right) v\left(y-y_{n, k}\right)} H(u, v) d u d v \\
& \quad=T_{x} T_{y} \int_{-\Omega_{y}}^{+\Omega_{y}} \int_{-\Omega_{x}}^{+\Omega_{x}} e^{j\left(1 / b_{1}\right) u\left(x-x_{l}\right)} e^{\left(1 / b_{2}\right) v\left(y-y_{n, k}\right)} d u d v \\
& \quad=T_{x} T_{y} \frac{2 \sin \left[\Omega_{x}\left(x-x_{n, k}\right) / b_{1}\right]}{\left(x-x_{n, k}\right) / b_{1}} \frac{2 \sin \left[\Omega_{y}\left(y-y_{n, k}\right) / b_{2}\right]}{\left(y-y_{n, k}\right) / b_{2}} .
\end{aligned}
$$

Substitute (3.8) into (3.7) we obtain

$$
\begin{aligned}
f(x, y)= & e^{-\left(a_{1} / 2 b_{1}\right) x^{2}-\left(a_{2} / 2 b_{2}\right) y^{2}} \\
& \times \iint_{-\infty}^{+\infty} f\left(x_{n, k}, y_{n, k}\right) e^{\left(a_{1} / 2 b_{1}\right) x_{n, k^{2}}} e^{\left(a_{2} / 2 b_{2}\right) y_{n, k^{2}}} \\
& \times \frac{T_{x} \sin \left[\Omega_{x}\left(x-x_{n, k}\right) / b_{1}\right]}{\pi\left(x-x_{n, k}\right)} \frac{T_{y} \sin \left[\Omega_{y}\left(y-y_{n, k}\right) / b_{2}\right]}{\pi\left(y-y_{n, k}\right)} d x_{n, k} d y_{n, k} .
\end{aligned}
$$

The final result can be obtained by substituting (3.1) into above equation and letting the sample interval to be $T_{x}=\pi b_{x} / \Omega_{x}, T_{y}=\pi b_{y} / \Omega_{y}$.

\subsection{Special Cases of the Uniform Sampling Theorem of Two-Dimensional Signals}

(1) Let $A=\left(\begin{array}{cc}0 & 1 \\ -1 & 0\end{array}\right)$ and $B=\left(\begin{array}{cc}0 & 1 \\ -1 & 0\end{array}\right)$, then (3.2) and (3.6) reduce to

$$
\begin{gathered}
D L_{f}^{A, B}(u, v)=\frac{1}{T_{x} T_{y}} \sum_{k=-\infty}^{+\infty} \sum_{l=-\infty}^{+\infty} L_{f}^{A, B}\left(u-\frac{k 2 \pi}{T_{x}}, v-\frac{l 2 \pi}{T_{y}}\right) \\
f(x, y)=\sum_{n=-\infty}^{+\infty} \sum_{k=-\infty}^{+\infty} f\left(n T_{x}, k T_{y}\right) \frac{\sin \left[\Omega_{x}\left(x-n T_{x}\right)\right]}{\Omega_{x}\left(x-n T_{x}\right)} \frac{\sin \left[\Omega_{y}\left(y-k T_{y}\right)\right]}{\Omega_{y}\left(y-k T_{y}\right)}
\end{gathered}
$$

which are the well known digital spectral representation and the reconstruction formula of uniformly sampled two-dimensional signals in traditional Fourier transform domain. 
(2) Let $A=\left(\begin{array}{cc}\cos \theta_{1} & \sin \theta_{1} \\ -\sin \theta_{1} & \cos \theta_{1}\end{array}\right)$ and $B=\left(\begin{array}{cc}\cos \theta_{2} & \sin \theta_{2} \\ -\sin \theta_{2} & \cos \theta_{2}\end{array}\right)$, then (3.2) and (3.6) reduce to

$$
\begin{aligned}
D L_{f}^{A, B}(u, v)= & \frac{1}{T_{x} T_{y}} e^{j\left[\left(\cot \theta_{1} u^{2} / 2\right)+\left(\cot \theta_{2} v^{2} / 2\right)\right]} \\
& \times \sum_{k=-\infty}^{+\infty} \sum_{l=-\infty}^{+\infty} L_{f}^{A, B}\left(u-\frac{2 \pi k}{T_{x}} \sin \theta_{1}, v-\frac{2 \pi l}{T_{y}} \sin \theta_{2}\right) \\
& \times e^{-j\left(\cot \theta_{1} / 2\right)\left(u-\left(2 \pi k / T_{x}\right) \sin \theta_{1}\right)^{2}} e^{-j\left(\cot \theta_{2} / 2\right)\left(v-\left(2 \pi l / T_{y}\right) \sin \theta_{2}\right)^{2}}, \\
f(x, y)= & e^{-j\left(\cot \theta_{1} / 2\right) x^{2}-j\left(\cot \theta_{2} / 2\right) y^{2}} \\
& \times \sum_{n=-\infty}^{+\infty} \sum_{k=-\infty}^{+\infty} f\left(n T_{x}, k T_{y}\right) e^{j\left(\cot \theta_{1} / 2\right)\left(n T_{x}\right)^{2}+j\left(\cot \theta_{2} / 2\right)\left(k T_{y}\right)^{2}} \\
& \cdot \frac{\sin \left[\csc \theta_{1} \Omega_{x}\left(x-n T_{x}\right)\right]}{\csc \theta_{1} \Omega_{x}\left(x-n T_{x}\right)} \frac{\sin \left[\csc \theta_{2} \Omega_{y}\left(y-k T_{y}\right)\right]}{\csc \theta_{2} \Omega_{y}\left(y-k T_{y}\right)} .
\end{aligned}
$$

They can be looked as the digital spectrum of a uniformly sampled signal and the reconstruction formula in fractional Fourier transform domain, respectively.

\section{The Spectral Analysis of Nonuniformly Sampled Signals}

It is shown in the preliminary Section that the recurrent nonuniform sampling occurs frequently in real applications, and there are many published works on the spectral analysis methods in the Fourier domain [4-6]. However, these results are only suitable for the bandlimited signals in the Fourier domain and may obtain incorrect results for nonbanlimited signals of Fourier domain [19]. Because the nonbandlimited signals can be bandlimited in the LCT domain [8], so it is worthwhile and interesting to investigate the spectral analysis problems in the LCT domain.

\subsection{Spectral Analysis of One-Dimensional Signal from Recurrent Nonuniform Samples}

In above-mentioned, one-dimensional periodic nonuniform sampling model, the $m$ th sampling sequence can be seen as uniformly sampling the signal with sampling period MT:

$$
s_{m}=\left[f\left(t_{m}\right), f\left(t_{M+m}\right), f\left(t_{2 M+m}\right), \ldots\right],
$$

the total sampling sequence can be represented as:

$$
s=\left\{f\left(t_{k m}\right) \mid t_{k m}=k M T+t_{m}, m=0,1,2, \ldots, M-1, k \in Z\right\} .
$$


Let $\bar{s}_{m}=\left[f\left(t_{m}\right), 0, \ldots,(M-1)\right.$ zeros, $\left.f\left(t_{M+m}\right), 0,0, \ldots\right], m=0,1, \ldots, M-1$ and shift $\bar{s}_{m} m T$ positions to the right, for $m=0,1, \ldots, M-1$ to obtain

$$
\bar{s}_{m} z^{-m}=\left[(m \text { zeros }) f\left(t_{m}\right), 0, \ldots,(M-1) \text { zeros, } f\left(t_{M+m}\right), 0,0, \ldots\right],
$$

where $z^{-1}$ is the unit delay operator. Finally, summing up all the subsequences to obtain the original sequence $s=\sum_{m=0}^{M-1} \bar{s}_{m} z^{-m}$, therefore the discrete LCT of the periodic nonuniformly sampled signal sequence $s$ can be derived by the summation of the LCT of $M$ subsequence $\bar{s}_{m} z^{-m}(m=0$ to $M-1)$. The result can be represented as the following theorem.

Theorem 4.1. Let $f(t)$ be an analog signal with its CLCT $L_{f}^{A}(u)$ bandlimited to $\left(-\Omega_{\alpha}, \Omega_{\alpha}\right)$ in the LCT domain; $f(t)$ is sampled nonuniformly with recurrent period MT. Then, the digital spectrum of these periodic nonuniformly sampled points can be represented as:

$$
\begin{aligned}
D L_{f}^{A}[f(t)](u)= & \frac{1}{M T} \times \sum_{n=-\infty}^{\infty} \sum_{m=0}^{M-1} L_{f}^{A}\left[u-\frac{2 \pi n}{N T} b+a r_{m} T\right] \\
& \cdot e^{j(1 / 2)\left(\left(a-a^{2} d\right) / b\right) T^{2} r_{m}^{2}} e^{j(2 \pi n / M T) d(u-(\pi n / M T) b)} \\
& \cdot e^{-j[((1-a d) / b) u-(1-a d)(2 \pi n / M T)] r_{m} T} e^{-j(2 \pi n m / M)},
\end{aligned}
$$

where $T$ is the nominal uniform sampling period, and $r_{m}=\left(m T-t_{m}\right) / T, m=0,1, \ldots, M-1$ are sampling time offsets.

Proof. The result can be derived by using the similar methods as in [5] and the properties of LCT.

\subsection{The Spectral Analysis of Two-Dimensional Signal}

Firstly, consider the function $f_{s}(x, y)$ which are obtained by sampling a two-dimensional signal $f(x, y)$ at nonuniformly spaced grids. The sampling offsets on both $X$-axis and $Y$-axis are periodic and have the period $M$ and $N$, respectively. That means

$$
x_{u, v}=u T_{x}+\Delta x_{u, v}, \quad y_{u, v}=v T_{y}+\Delta y_{u, v}
$$

where $T_{x}$ and $T_{y}$ are nominal uniform sampling periods on the $X$-axis and $Y$-axis, respectively. $\Delta x_{u, v}$ and $\Delta y_{u, v}$ are periodic sequence with period $M$ on $u$ and $N$ on $v$. Because of the periodic structure of $\Delta x_{u, v}$ and $\Delta y_{u, v}$, we can let $u=k M+m, v=l N+n$, where $-\infty<k, l<\infty, 0 \leq m \leq M-1$ and $0 \leq n \leq N-1$. Then

$$
\begin{aligned}
x_{u, v} & =u T_{x}+\Delta x_{u, v}=(k M+m) T_{x}+\Delta x_{k M+m, l N+n} \\
& =k M T_{x}+m T_{x}+\Delta x_{m, n}=k M T_{x}+m T_{x}+r_{m, n} T_{x}, \\
y_{u, v} & =v T_{y}+\Delta y_{u, v}=(l N+n) T_{y}+\Delta y_{k M+m, l N+n} \\
& =l N T_{y}+n T_{y}+\Delta y_{m, n}=l N T_{y}+n T_{y}+s_{m, n} T_{y},
\end{aligned}
$$


where $r_{m, n}=\Delta x_{m, n} / T_{x}$ and $s_{m, n}=\Delta y_{m, n} / T_{y}$ are the ratios of sampling offsets to the nominal uniform sampling periods. The sampling signal $f_{s}(x, y)$ can be represented as:

$$
f_{s}(x, y)=f(x, y) \times \delta\left[x-\left(M k+m+r_{m, n}\right) T_{x}\right] \times \delta\left[y-\left(N l+n+s_{m, n}\right) T_{y}\right]
$$

The DLCT of this two-dimensional signal $f\left(x_{u, v}, y_{u, v}\right)$ can be obtained as:

$$
\begin{aligned}
D L_{f}^{A, B}\left(u^{\prime}, v^{\prime}\right)= & \sum_{u=-\infty}^{+\infty} \sum_{v=-\infty}^{+\infty} f\left(x_{u, v}, y_{u, v}\right) \\
& \times \frac{1}{2 \pi j} \sqrt{\frac{1}{b_{1} b_{2}}} e^{j\left[\left(d_{1} u^{\prime 2} / 2 b_{1}\right)+\left(d_{2} v^{\prime 2} / 2 b_{2}\right)\right]} e^{-j\left[u^{\prime} u T_{x} / b_{1}+v^{\prime} v T_{y} / b_{2}\right]} \\
& \times e^{j\left[a_{1}\left(u T_{x}\right)^{2} / 2 b_{1}+a_{2}\left(v T_{y}\right)^{2} / 2 b_{2}\right]},
\end{aligned}
$$

where $b_{1} b_{2} \neq 0,|A|=|B|=1$.

Based on the properties of the DLCT and (4.8), the digital spectrum of the nonuniformly sampled two-dimensional signals can be represented in the following Theorem 4.2.

Theorem 4.2. Let $f(x, y)$ be an analog two-dimensional signal with its continuous LCT bandlimited in the LCT domain, $f(x, y)$ is sampled periodically with period $M$ and $N$ in the $x$ and $y$ directions. Then the digital spectrum of these periodic nonuniformly sampled points can be presented as:

$$
\begin{aligned}
D L_{f}^{A, B}\left(u^{\prime}, v^{\prime}\right)= & \frac{1}{T_{x} T_{y}} e^{j\left[\left(d_{1} u^{\prime 2} / 2 b_{1}\right)+\left(d_{2} v^{\prime 2} / 2 b_{2}\right)\right]} \\
& \times \sum_{k=-\infty}^{+\infty} \sum_{l=-\infty}^{+\infty} H\left(k, l, u^{\prime}, v^{\prime}\right) \\
& \times e^{-j\left[\left(d_{1} / 2 b_{1}\right)\left(u^{\prime}+a_{1} r_{m, n} T_{x}-\left(2 \pi k / M T_{x}\right) b_{1}\right)^{2}+\left(d_{2} / 2 b_{2}\right)\left(v^{\prime}+a_{2} s_{m, n} T_{y}-\left(2 \pi l / N T_{y}\right) b_{2}\right)^{2}\right]} \\
& \cdot L_{f}^{A, B}\left(u^{\prime}+a_{1} r_{m, n} T_{x}-\frac{2 \pi k}{M T_{x}} b_{1}, v^{\prime}+a_{2} s_{m, n} T_{y}-\frac{2 \pi l}{N T_{y}} b_{2}\right)
\end{aligned}
$$

where

$$
\begin{aligned}
H\left(k, l, u^{\prime}, v^{\prime}\right)= & \left(\frac{1}{M N}\right) \times \sum_{m=0}^{M-1} \sum_{n=0}^{N-1} e^{j\left[\left(u^{\prime} / b_{1}+a_{1} r_{m, n} T_{x} / 2 b_{1}\right) r_{m, n} T_{x}-\left(2 \pi k\left(m+r_{m, n}\right) / M\right)\right]} \\
& \times e^{j\left[\left(v^{\prime} / b_{2}+a_{2} s_{m, n} T_{y} / 2 b_{2}\right) s_{m, n} T_{y}-\left(2 \pi l\left(n+s_{m, n}\right) / N\right)\right]} .
\end{aligned}
$$


Proof. Following the definition of the DLCT, the digital spectrum of the samples can be written as:

$$
\begin{aligned}
D L_{f}^{A, B}\left(u^{\prime}, v^{\prime}\right)= & \sum_{u=-\infty}^{+\infty} \sum_{v=-\infty}^{+\infty} f_{s}(x, y) \frac{1}{2 \pi j} \sqrt{\frac{1}{b_{1} b_{2}}} \times e^{j\left[\left(d_{1} u^{\prime 2} / 2 b_{1}\right)+\left(d_{2} v^{\prime 2} / 2 b_{2}\right)\right]} \\
& \times e^{-j\left[u^{\prime} u T_{x} / b_{1}+v^{\prime} v T_{y} / b_{2}\right]} \times e^{j\left[a_{1}\left(u T_{x}\right)^{2} / 2 b_{1}+a_{2}\left(v T_{y}\right)^{2} / 2 b_{2}\right]} \\
= & \sum_{k=-\infty}^{+\infty} \sum_{l=-\infty}^{+\infty} \sum_{m=0}^{M-1 N-1} \sum_{n=0} \frac{1}{2 \pi j} \sqrt{\frac{1}{b_{1} b_{2}}} \cdot e^{j\left[\left(d_{1} u^{\prime 2} / 2 b_{1}\right)+\left(d_{2} v^{\prime 2} / 2 b_{2}\right)\right]} \\
& \cdot e^{-j\left[u^{\prime}(k M+m) T_{x} / b_{1}+v^{\prime}(l N+n) T_{y} / b_{2}\right]} \\
& \cdot e^{j\left[a_{1}(k M+m)^{2} T_{x}{ }^{2} / 2 b_{1}+a_{2}(l N+n)^{2} T_{y}{ }^{2} / 2 b_{2}\right]} \\
& \times \iint_{-\infty}^{+\infty} f(x, y) \times \delta\left[x-\left(M k+m+r_{m, n}\right) T_{x}\right] \times \delta\left[y-\left(N l+n+s_{m, n}\right) T_{y}\right] d x d y .
\end{aligned}
$$

By the Fourier series of $\delta\left[x-\left(M k+m+r_{m, n}\right) T_{x}\right]$ and $\delta\left[y-\left(N l+n+s_{m, n}\right) T_{y}\right],(4.11)$ can be rearranged as:

$$
\begin{aligned}
D L_{f}^{A, B}\left(u^{\prime}, v^{\prime}\right)= & \frac{1}{2 \pi j} \sqrt{\frac{1}{b_{1} b_{2}}} \cdot e^{j\left[\left(d_{1} u^{\prime 2} / 2 b_{1}\right)+\left(d_{2} v^{\prime 2} / 2 b_{2}\right)\right]} \\
& \cdot \sum_{m=0}^{M-1} \sum_{n=0}^{N-1} \iint_{-\infty}^{+\infty} f(x, y) \times e^{j\left[u^{\prime} r_{m, n} T_{x} / b_{1}+v^{\prime} s_{m, n} T_{y} / b_{2}\right]} \\
& \cdot e^{j\left[a_{1}\left(r_{m, n} T_{x}\right)^{2} / 2 b_{1}+a_{2}\left(s_{m, n} T_{y}\right)^{2} / 2 b_{2}\right]} \\
& \times e^{-j\left[u^{\prime} x / b_{1}+v^{\prime} y / b_{2}\right]} e^{j\left[a_{1}\left(x^{2}-2 r_{m, n} T_{x} x\right) / 2 b_{1}+a_{2}\left(y^{2}-2 s_{m, n} T_{y} y\right) / 2 b_{2}\right]} \\
& \times\left\{\frac{1}{M T_{x}} \sum_{k=-\infty}^{+\infty} e^{j 2 \pi k\left[x-\left(m+r_{m, n}\right) T_{x}\right] / M T_{x}} \times \frac{1}{N T_{y}} \sum_{l=-\infty}^{+\infty} e^{j 2 \pi l\left[y-\left(n+s_{m, n}\right) T_{y}\right] / N T_{y}}\right\} d x d y .
\end{aligned}
$$

The final result can be obtained by rearranging (4.12) and following the definition of the LCT.

\subsection{Special Cases of the Derived Results}

Before introducing the new results, let us investigate the special cases of the derived results. 
(1) When the parameters of LCT reduce to $A=\left(\begin{array}{cc}0 & 1 \\ -1 & 0\end{array}\right)$ and $B=\left(\begin{array}{cc}0 & 1 \\ -1 & 0\end{array}\right),(4.9)$ can be reduced to

$$
D L_{f}^{A, B}\left(u^{\prime}, v^{\prime}\right)=\frac{1}{T_{x} T_{y}} \sum_{k=-\infty}^{+\infty} \sum_{l=-\infty}^{+\infty} H\left(k, l, u^{\prime}, v^{\prime}\right) L_{f}^{A, B}\left(u^{\prime}-\frac{k 2 \pi}{M T_{x}}, v^{\prime}-\frac{l 2 \pi}{N T_{y}}\right),
$$

where

$$
\begin{aligned}
H\left(k, l, u^{\prime}, v^{\prime}\right)= & \left(\frac{1}{M N}\right) \sum_{m=0}^{M-1} \sum_{n=0}^{N-1} e^{j\left[\left(u^{\prime}+\left(k 2 \pi / M T_{x}\right)\right) r_{m, n} T_{x}-m k(2 \pi / M)\right]} \\
& \times e^{j\left[\left(v^{\prime}+\left(l 2 \pi / N T_{y}\right)\right) s_{m, n} T_{y}-n l(2 \pi / N)\right]},
\end{aligned}
$$

which is the well known digital spectrum of periodic nonuniformly sampled two-dimensional signal in Fourier transform domain [6].

(2) Furthermore, when $r_{m, n}$ and $s_{m, n}$ are zeros, In this case, the periodic nonuniform sampling model reduces to uniform sampling model. It is easy to verify that $H\left(k, l, u^{\prime}, v^{\prime}\right)=1$ when $k$ is an integer multiple of $M$ and $l$ is an integer multiple of $N$; otherwise $H\left(k, l, u^{\prime}, v^{\prime}\right)=$ 0 . Therefore,

$$
\begin{aligned}
D L_{f}^{A, B}(u, v)= & \frac{1}{T_{x} T_{y}} e^{j\left[\left(d_{1} u^{2} / 2 b_{1}\right)+\left(d_{2} v^{2} / 2 b_{2}\right)\right]} \\
& \times \sum_{k=-\infty}^{+\infty} \sum_{l=-\infty}^{+\infty} L_{f}^{A, B}\left(u-\frac{2 \pi k}{T_{x}} b_{1}, v-\frac{2 \pi l}{T_{y}} b_{2}\right) e^{-j\left(d_{1} / 2 b_{1}\right)\left(u-\left(2 \pi k / T_{x}\right) b_{1}\right)^{2}} \\
& \times e^{-j\left(d_{2} / 2 b_{2}\right)\left(v-\left(2 \pi k / T_{x}\right) b_{2}\right)^{2}}
\end{aligned}
$$

which is the digital spectrum of a uniformly sampled two-dimensional signal in linear canonical transform domain derived in Section 3 (Theorem 3.1).

(3) When the parameter of LCT reduces to $A=\left(\begin{array}{cc}\cos \theta_{1} & \sin \theta_{1} \\ -\sin \theta_{1} & \cos \theta_{1}\end{array}\right)$ and $B=\left(\begin{array}{cc}\cos \theta_{2} & \sin \theta_{2} \\ -\sin \theta_{2} & \cos \theta_{2}\end{array}\right)$, then (4.6) reduces to

$$
\begin{aligned}
D L_{f}^{A, B}\left(u^{\prime}, v^{\prime}\right)= & \frac{1}{T_{x} T_{y}} e^{j\left[\left(d_{1} u^{\prime 2} / 2 b_{1}\right)+\left(d_{2} v^{\prime 2} / 2 b_{2}\right)\right]} \\
& \times \sum_{k=-\infty}^{+\infty} \sum_{l=-\infty}^{+\infty} H\left(k, l, u^{\prime}, v^{\prime}\right) \\
& \times e^{-j\left[\left(d_{1} / 2 b_{1}\right)\left(u^{\prime}+a_{1} r_{m, n} T_{x}-\left(2 \pi k / M T_{x}\right) b_{1}\right)^{2}+\left(d_{2} / 2 b_{2}\right)\left(v^{\prime}+a_{2} s_{m, n} T_{y}-\left(2 \pi l / N T_{y}\right) b_{2}\right)^{2}\right]} \\
& \times L_{f}^{A, B}\left(u^{\prime}+a_{1} r_{m, n} T_{x}-\frac{2 \pi k}{M T_{x}} b_{1}, v^{\prime}+a_{2} S_{m, n} T_{y}-\frac{2 \pi l}{N T_{y}} b_{2}\right)
\end{aligned}
$$


where

$$
\begin{aligned}
H\left(k, l, u^{\prime}, v^{\prime}\right)= & \left(\frac{1}{M N}\right) \times \sum_{m=0}^{M-1} \sum_{n=0}^{N-1} e^{j\left[\left(u^{\prime} \csc \theta_{1}+\cot \theta_{1} r_{m, n} T_{x} / 2+\left(k 2 \pi / M T_{x}\right)\right) r_{m, n} T_{x}-m k(2 \pi / M)\right]} \\
& \times e^{j\left[\left(v^{\prime} \csc \theta_{2}+\cot \theta_{2} s_{m, n} T_{y} / 2+\left(l 2 \pi / N T_{y}\right)\right) s_{m, n} T_{y}-n l(2 \pi / N)\right]},
\end{aligned}
$$

this result can be looked as the digital spectral of two-dimensional signals in the fractional Fourier domain.

(4) For one-dimensional case, when the parameter of LCT reduces to $A=\left(\begin{array}{c}\cos \theta \\ \sin \theta \\ -\sin \theta \cos \theta\end{array}\right)$, the results of Theorem 4.1 reduce to the results of fractional Fourier transform domain of [24], and reduce to the results of Fourier domain of [5] when $\theta=\pi / 2$.

From the above-mentioned special cases of the Theorem 4.2, we conclude that the formula we derived is more general and more suitable for nonstationary signal processing. Because of chirp signal is one of the mostly used nonstationary signal in the Radar signal processing society. Therefore, the properties and potential applications of this result for chirp signal processing are investigated in the following Sections.

\subsection{The Spectrum of Chirp Signals in the LCT Domain}

The chirp signal can be looked as one of the typical nonstationary signals in modern signal processing community. It is shown that the LCT is one of the most important tools for chirp signal processing. It is therefore worthwhile and interesting to investigate the spectrum of chirp signal in the LCT domain. It is easy to derive that when $\left(a_{1} / b_{1}\right)+m_{1}=0$, $\left(a_{2} / b_{2}\right)+m_{2}=0$, the continuous LCT of a two-dimensional chirp signal $f(x, y)=$ $C e^{j\left(\omega_{1} x+(1 / 2) m_{1} x^{2}\right)} e^{j\left(\omega_{2} y+(1 / 2) m_{2} y^{2}\right)}$ can be obtained as:

$$
L_{f}^{A, B}(u, v)=\frac{C}{2 \pi j} \sqrt{\frac{1}{b_{1} b_{2}}} e^{j\left[\left(d_{1} u^{2} / 2 b_{1}\right)+d_{2} v^{2} / 2 b_{2}\right]} \cdot \delta\left(\omega_{1}-\frac{u}{b_{1}}\right) \cdot \delta\left(\omega_{2}-\frac{v}{b_{2}}\right) .
$$

Substitute (4.18) in (4.9), the digital spectrum of two-dimensional chirp signal can be represented as following theorem.

Theorem 4.3. Let $f(x, y)=C e^{j\left(\omega_{1} x+(1 / 2) m_{1} x^{2}\right)} e^{j\left(\omega_{2} y+(1 / 2) m_{2} y^{2}\right)}$, then the digital spectrum from the periodic nonuniformly sampled signal points with period $M$ and $N$ in the $x$ and $y$ directions, respectively, can be represented by the following:

$$
\begin{aligned}
D L_{f}^{A, B}\left(u^{\prime}, v^{\prime}\right)= & \frac{C}{\sqrt{2 \pi j}} \sqrt{\frac{1}{b_{1} b_{2}}} \frac{1}{T_{x} T_{y}} e^{j\left[\left(d_{1} u^{\prime 2} / 2 b_{1}\right)+\left(d_{2} v^{\prime 2} / 2 b_{2}\right)\right]} \sum_{k=-\infty}^{+\infty} \sum_{l=-\infty}^{+\infty} H(k, l) \\
& \cdot \delta\left[\omega_{1}-\left(\frac{u^{\prime}}{b_{1}}+\frac{a_{1}}{b_{1}} r_{m, n} T_{x}-\frac{2 \pi k}{M T_{x}}\right)\right] \delta\left[\omega_{2}-\left(\frac{v^{\prime}}{b_{2}}+\frac{a_{2}}{b_{2}} s_{m, n} T_{y}-\frac{2 \pi l}{N T_{y}}\right)\right],
\end{aligned}
$$




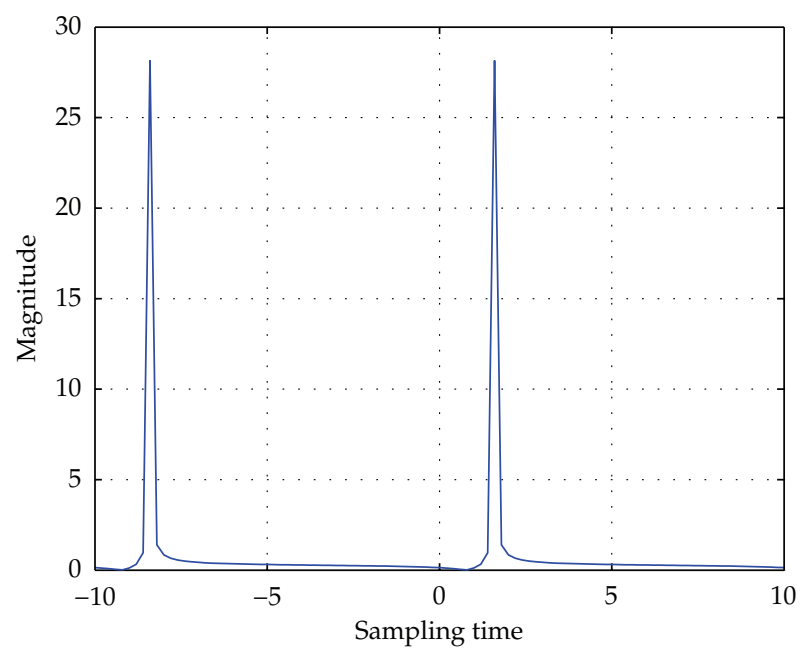

Figure 2: The spectrum of uniform sampling points.

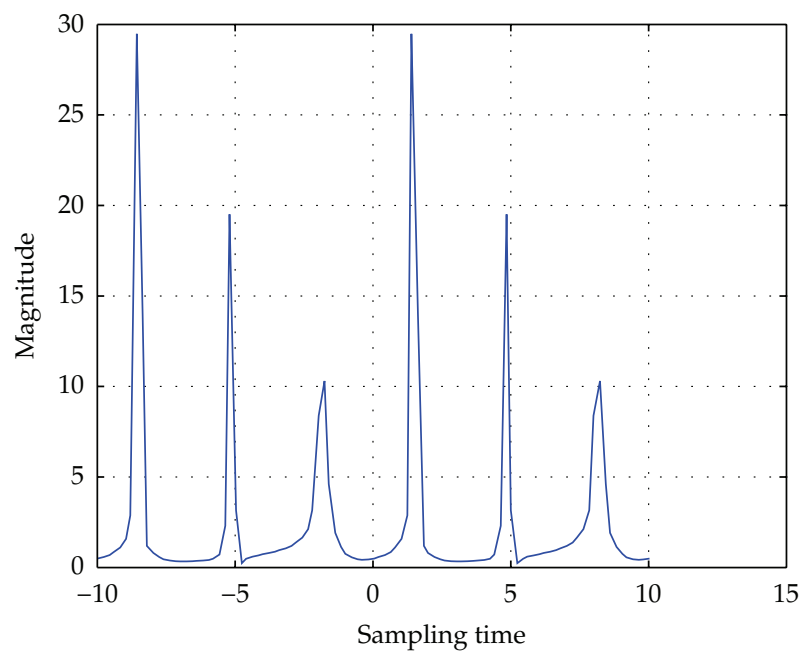

Figure 3: The spectrum of nonuniform sampling points with $M=3$.

where

$$
\begin{aligned}
H(k, l)= & \frac{1}{M N} \times \sum_{m=0}^{M-1} \sum_{n=0}^{N-1} e^{j\left[\left(\omega_{1}-\left(a_{1} / 2 b_{1}\right) r_{m, n} T_{x}+\left(4 \pi k / M T_{x}\right)\right) r_{m, n} T_{x}-m k(2 \pi / M)\right]} \\
& \times e^{j\left[\left(\omega_{2}-\left(a_{2} / 2 b_{2}\right) s_{m, n} T_{y}+\left(4 \pi l / M T_{y}\right)\right) s_{m, n} T_{y}-n l(2 \pi / N)\right]} .
\end{aligned}
$$

Proof. This formula can be derived by substituting (4.18) in (4.9), and applying the properties of the discrete Fourier transform.

This theorem indicates that the LCT of a two-dimensional chirp signal from periodic nonuniform sampling points contains line spectra components in the LCT domain, with 


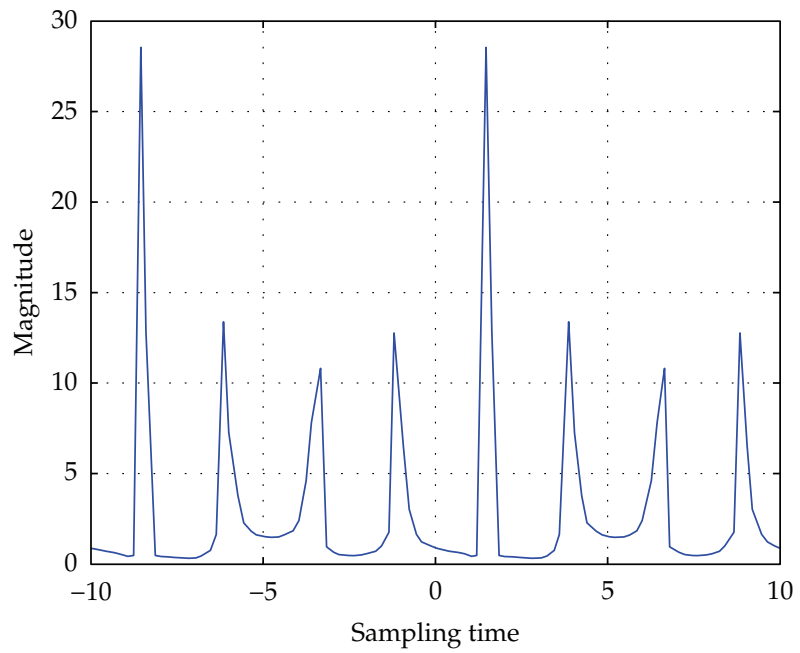

Figure 4: The spectrum of nonuniform sampling points with $M=4$.

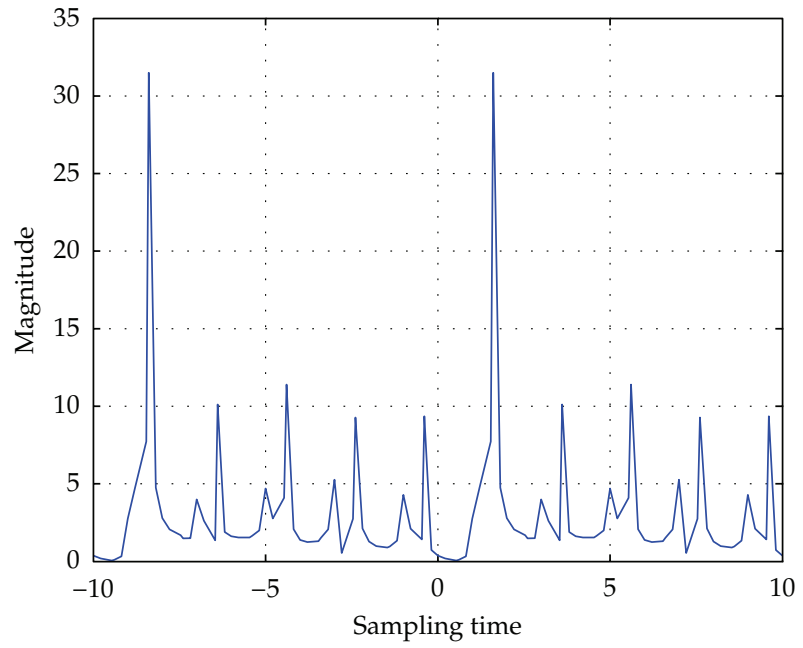

Figure 5: The spectrum of nonuniform sampling points with $M=5$.

the magnitude at $(k, l)$ being $H(k, l)$. The difference between the uniform and periodic nonuniform sampling points is that for periodic nonuniform sampling signals, it will produce some additional pseudo spectra in the same LCT domain besides the main spectral component.

\subsection{The Simulation Results}

In order to show the importance of spectrum analysis of the sampling points associated with the LCT, we choose the recurrent nonuniform sampling model as an example to perform the simulation in the LCT domain. 


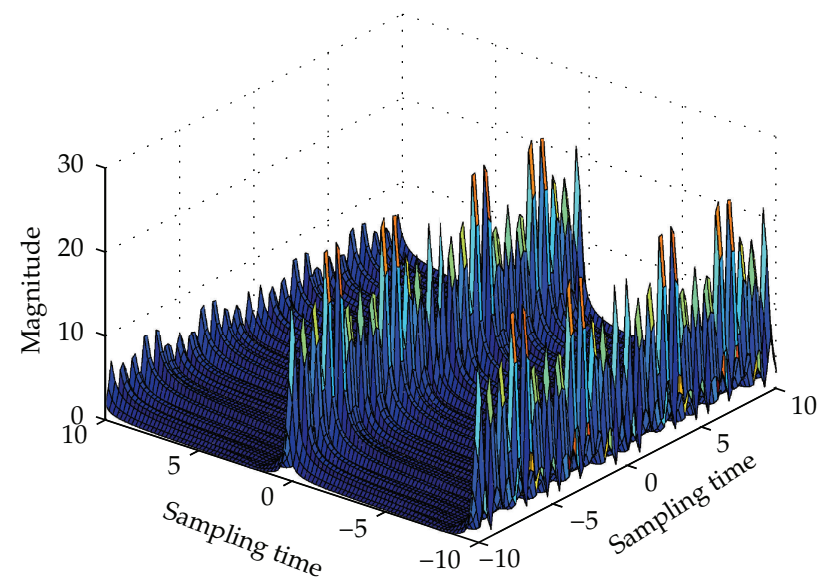

Figure 6: The spectrum of uniform sampling points.

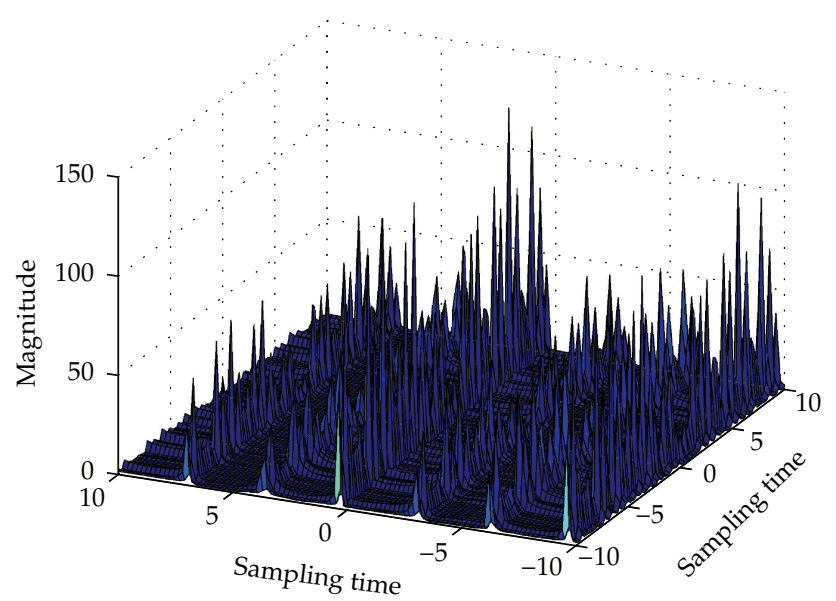

Figure 7: The spectral of nonuniformly sampled signal with $N=4, M=3$.

Firstly, we choose the chirp signal as $f(t)=\exp \left\{j\left(5 t-6 t^{2}\right)\right\}$, the sampling period $T=0.2 \mathrm{~s}$ in a period $[-10,10]$. The spectral of uniformly sampled signal is plotted in Figure 2, it is shown that the uniform sampling in the time domain will produce the periodic spectrum in the LCT domain as reflected in Lemma 2.1. Figures 3, 4, and 5 plot the spectrum of nonuniformly sampling points for $M=3,4$, and 5, respectively. From these simulation results, we can see that it will produce additional $M-1$ pseudospectra in the same LCT domain besides the main spectral component. This was coinciding with the derived results shown in Theorem 4.1.

Secondly, we choose the two-dimensional chirp signal as $f(x, y)=\exp \{j(5 x-$ $\left.\left.6 x^{2}\right)\right\} \exp \left\{j\left(y-y^{2}\right)\right\}$, the sampling period of $x$ and $y$ is equal and choose to be $T=0.2 \mathrm{~s}$ in a period $[-10,10]$. The spectral of uniformly sampled signal is plotted in Figure 6 , it is shown in the simulation that the spectra of two-dimensional uniform sampling signal in the LCT domain are periodic as reflected in Theorem 3.1 of the paper. The spectral of periodic nonuniformly sampled signal with $N=4, M=3$ is plotted in Figure 7 . 
From Figures 6 and 7, we can conclude that the LCT spectrum of periodic nonuniform sampling points for two-dimensional chirp signals will produce the pseudospectra in the same LCT domain besides the main spectral component, as reflected in Theorem 4.3 of the paper.

\section{Conclusion}

Based on the spectral properties of uniform and nonuniform samples in the LCT domain, this paper investigates the spectrum of one and two dimensional periodic nonuniformly sampled signals in the LCT domain. The digital spectral representation of one- and twodimensional periodic nonuniformly sampled signals in the LCT domain has been obtained, and a detailed analysis for chirp signals has been carried out. The derived results can be looked as the generalization of the traditional results in the Fourier domain and fractional Fourier transform domain and more suitable for the nonstationary signal analysis and processing. The future work direction will be the spectrum analysis of $N$ th order periodic nonuniform sampling [23] points and the spectrum reconstruction from the periodic or $N$ th order periodic nonuniform sampling signals in the LCT domain.

\section{Acknowledgment}

This work was supported by the National Natural Science Foundation of China (no. 60901058 and no. 61171195), and the Beijing Natural Science Foundation (no. 1102029).

\section{References}

[1] F. Marvasti, Nonuniform Sampling Theory and Practice, Kluwer Academic, New York, NY, USA, 2000.

[2] A. J. Jerri, "The Shannon sampling theorem-its various extensions and applications: a tutorial review," Proceedings of the IEEE, vol. 65, no. 11, pp. 1565-1598, 1977.

[3] M. Unser, "Sampling-50 years after Shannon," Proceedings of the IEEE, vol. 88, no. 4, pp. 569-587, 2000.

[4] A. Feuer and G. C. Goodwin, "Reconstruction of multidimensional bandlimited signals from nonuniform and generalized samples," IEEE Transactions on Signal Processing, vol. 53, no. 11, pp. 42734282, 2005.

[5] Y. C. Jenq, "Digital spectra of nonuniformly sampled signals: fundamentals and high-speed waveform digitizers," IEEE Transactions on Instrumentation and Measurement, vol. 37, no. 2, pp. 245-251, 1988.

[6] Y. C. Jenq and L. Cheng, "Digital spectrum of a nonuniformly sampled two-dimensional signal and its reconstruction," IEEE Transactions on Instrumentation and Measurement, vol. 54, no. 3, pp. 1180-1187, 2005.

[7] A. Stern, "Why is the linear canonical transform so little known?" in Proceedings of the 5th International Workshop on Information Optics (WIO '06), AIP Conference Proceedings, pp. 225-234, June 2006.

[8] R. Tao, B. Deng, and Y. Wang, Fractional Fourier Transform and its Applications, Tisinghua University Press, 2009.

[9] N. E. Huang, Z. Shen, S. R. Long et al., "The empirical mode decomposition and the Hubert spectrum for nonlinear and non-stationary time series analysis," Proceedings of the Royal Society A, vol. 454, no. 1971, pp. 903-995, 1998.

[10] A. Bultheel and H. Martínez-Sulbaran, "Recent developments in the theory of the fractional Fourier and linear canonical transforms," Bulletin of the Belgian Mathematical Society, vol. 13, no. 5, pp. 9711005, 2006.

[11] J. Zhao, R. Tao, Y.-L. Li, and Y. Wang, “Uncertainty principles for linear canonical transform," IEEE Transactions on Signal Processing, vol. 57, no. 7, pp. 2856-2858, 2009.

[12] A. Stern, "Uncertainty principles in linear canonical transform domains and some of their implications in optics," Journal of the Optical Society of America A, vol. 25, no. 3, pp. 647-652, 2008. 
[13] D. Wei and Y. Li, "A convolution and product theorem for the linear canonical transform," IEEE Signal Processing Letters, vol. 16, no. 10, pp. 853-856, 2009.

[14] Y. Fu and L. Li, "Generalized analytic signal associated with linear canonical transform," Optics Communications, vol. 281, no. 6, pp. 1468-1472, 2008

[15] B.-Z. Li, R. Tao, and Y. Wang, "Hilbert transform associated with the linear canonical transform," Acta Armamentarii, vol. 27, no. 5, pp. 827-830, 2006.

[16] X. Guanlei, W. Xiaotong, and X. Xiaogang, "Generalized Hilbert transform and its properties in 2D LCT domain," Signal Processing, vol. 89, no. 7, pp. 1395-1402, 2009.

[17] B.-Z. Li, R. Tao, T.-Z. Xu, and Y. Wang, "The Poisson sum formulae associated with the fractional Fourier transform," Signal Processing, vol. 89, no. 5, pp. 851-856, 2009.

[18] A. Stern, "Sampling of linear canonical transformed signals," Signal Processing, vol. 86, no. 7, pp. 1421-1425, 2006.

[19] R. Tao, B.-Z. Li, Y. Wang, and G. K. Aggrey, “On sampling of band-limited signals associated with the linear canonical transform," IEEE Transactions on Signal Processing, vol. 56, no. 11, pp. 5454-5464, 2008.

[20] B.-Z. Li, R. Tao, and Y. Wang, "New sampling formulae related to linear canonical transform," Signal Processing, vol. 87, no. 5, pp. 983-990, 2007.

[21] D. Wei, Q. Ran, and Y. Li, "Reconstruction of band-limited signals from multichannel and periodic nonuniform samples in the linear canonical transform domain," Optics Communications, vol. 284, no. 19, pp. 4307-4315, 2011.

[22] D. Wei, Q. Ran, and Y. Li, "Multichannel sampling expansion in the linear canonical transform domain and its application to superresolution," Optics Communications, vol. 284, no. 23, pp. 5424-5429, 2011.

[23] Y. C. Eldar and A. V. Oppenheim, "Filterbank reconstruction of bandlimited signals from nonuniform and generalized samples," IEEE Transactions on Signal Processing, vol. 48, no. 10, pp. 2864-2875, 2000.

[24] R. Tao, B.-Z. Li, and Y. Wang, "Spectral analysis and reconstruction for periodic nonuniformly sampled signals in fractional Fourier domain," IEEE Transactions on Signal Processing, vol. 55, no. 7, pp. 3541-3547, 2007. 


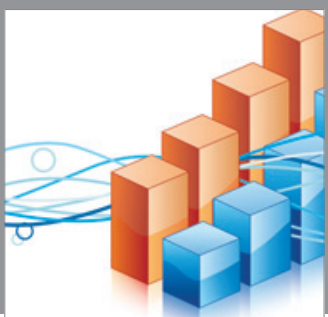

Advances in

Operations Research

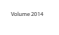

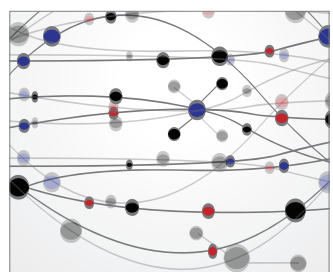

\section{The Scientific} World Journal
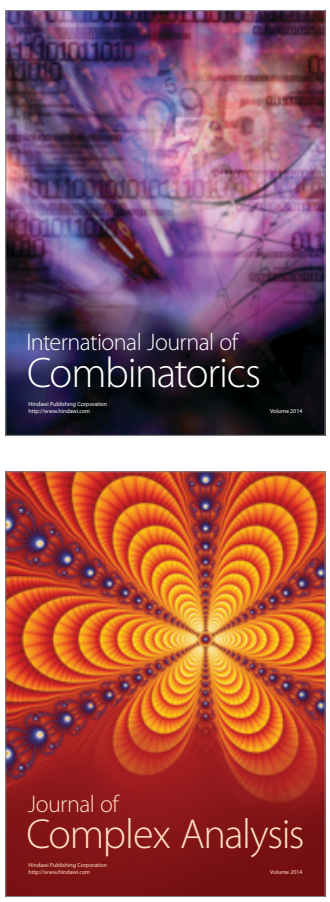

International Journal of

Mathematics and

Mathematical

Sciences
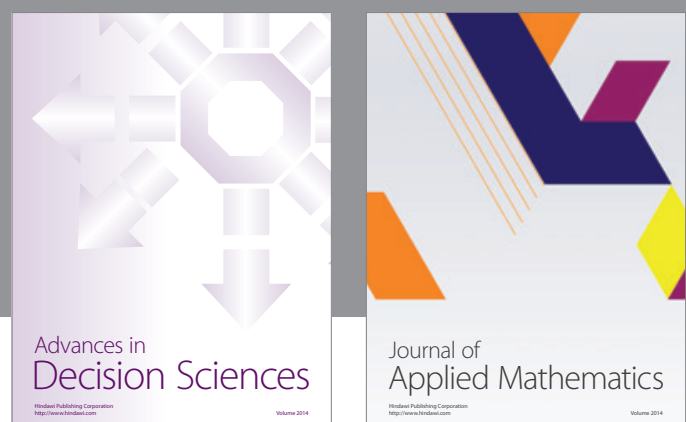

Journal of

Applied Mathematics
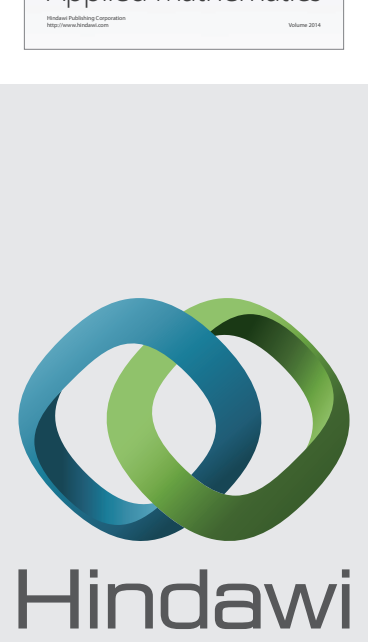

Submit your manuscripts at http://www.hindawi.com
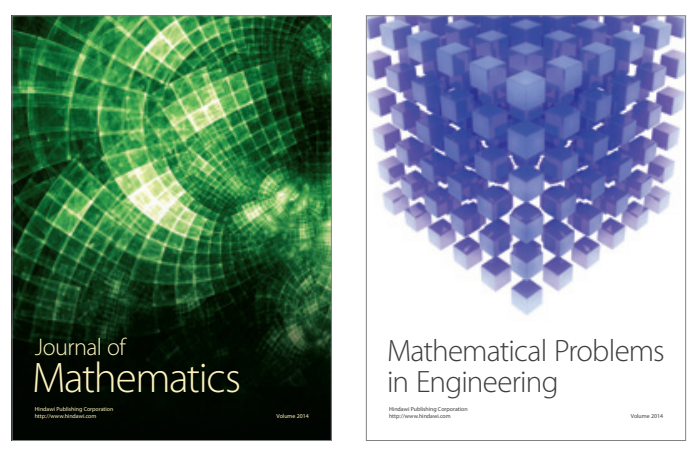

Mathematical Problems in Engineering
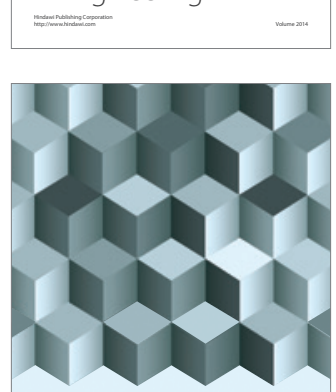

Journal of

Function Spaces
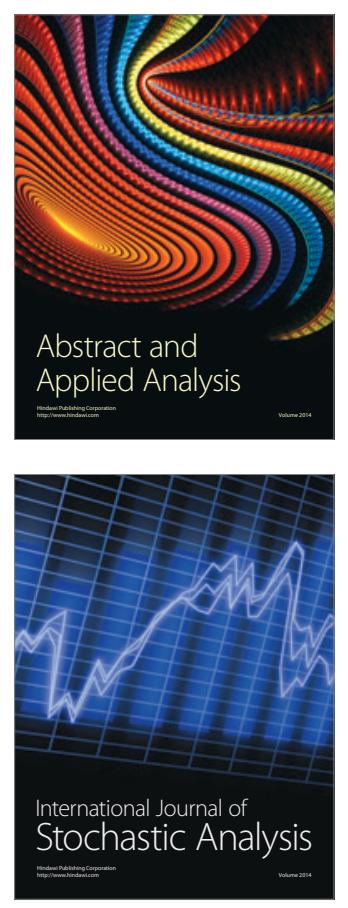

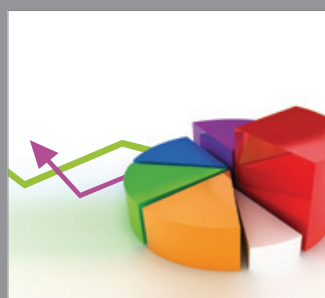

ournal of

Probability and Statistics

Promensencen
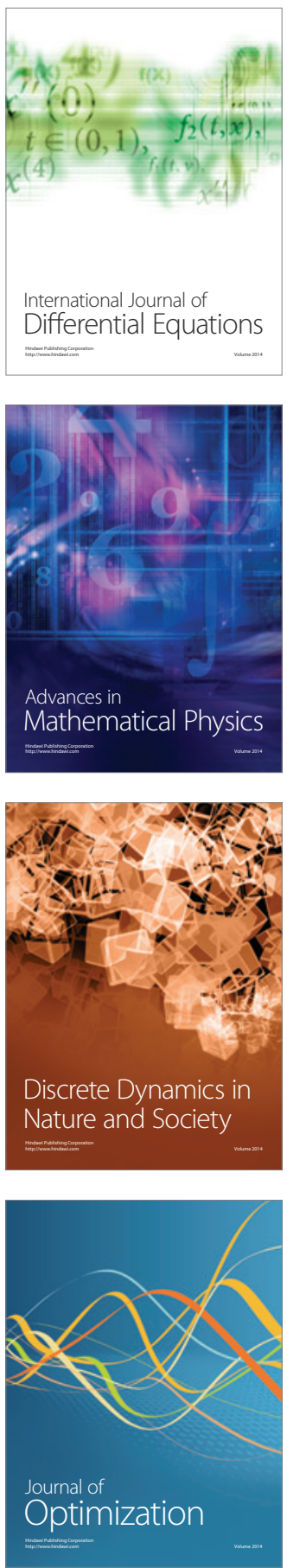